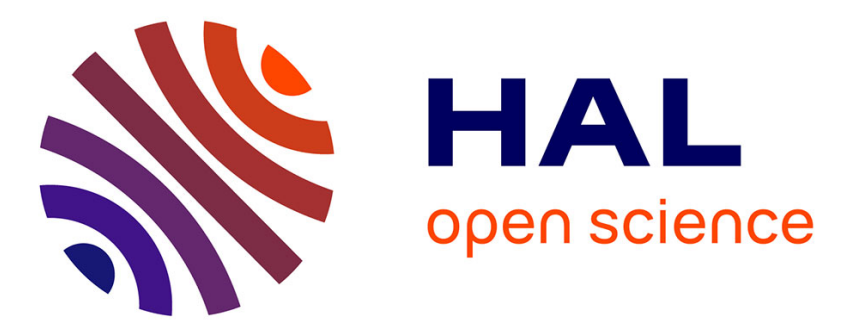

\title{
Characterization of HIFU transducers designed for sonochemistry application: Cavitation distribution and quantification
}

L. Hallez, F. Touyeras, J.-Y. Hihn, J. Klima, J.-L. Guey, M. Spajer, Y. Bailly

\section{- To cite this version:}

L. Hallez, F. Touyeras, J.-Y. Hihn, J. Klima, J.-L. Guey, et al.. Characterization of HIFU transducers designed for sonochemistry application: Cavitation distribution and quantification. International Congress on Ultrasonics 2009, Jan 2009, Santiago, Chile. pp.310-317, 10.1016/j.ultras.2009.09.011 . hal-00472207

\section{HAL Id: hal-00472207 \\ https://hal.science/hal-00472207}

Submitted on 23 Apr 2021

HAL is a multi-disciplinary open access archive for the deposit and dissemination of scientific research documents, whether they are published or not. The documents may come from teaching and research institutions in France or abroad, or from public or private research centers.
L'archive ouverte pluridisciplinaire HAL, est destinée au dépôt et à la diffusion de documents scientifiques de niveau recherche, publiés ou non, émanant des établissements d'enseignement et de recherche français ou étrangers, des laboratoires publics ou privés.

\section{(c)(1)}

Distributed under a Creative Commons Attribution| 4.0 International License 


\title{
Characterization of HIFU transducers designed for sonochemistry application: Cavitation distribution and quantification
}

\author{
L. Hallez ${ }^{\text {a }}$, F. Touyeras ${ }^{\text {a }}$, J.-Y. Hihn ${ }^{\text {a,* }}$, J. Klima ${ }^{\text {b }}$, J.-L. Guey ${ }^{\text {c }}$, M. Spajer ${ }^{\text {d }}$, Y. Bailly ${ }^{\text {d }}$ \\ ${ }^{a}$ Institut UTINAM/SRS, University of Franche-Comté UMR CNRS 6213, Besançon, France \\ ${ }^{\mathrm{b}}$ Institut Heyrovsky of Physical Chemistry, Prague, Czech Republic \\ ' IMASONIC Company, Voray sur l'Ognon, France \\ ${ }^{\mathrm{d}}$ Institut FEMTO-ST, University of Franche-Comté UMR CNRS 6174, Besançon, France
}

Acoustic field distribution was determined in HIFU sonoreactors as well as localization of cavitation activ-ity by crossing different techniques: modeling, hydrophone measurements, laser tomography and SCL measurements. Particular care was taken with quantification of this last technique by pixels or photon counting. Cavitation bubbles generated by HIFU are mainly located on the outer layer of the propagation cone in the post-focal zone. Greatest acoustic activity is not located at the geometrical focal, but corre-sponds to a high concentration of bubbles zone. On the contrary, the main sonochemical activity shifts slightly toward the transducer, whereas quenching of inertial cavitation is observed directly at the focal. Finally, SCL thresholds have been determined.

\section{Introduction}

Even if there is considerable literature concerning high frequency transducers, most of the papers dealing with HIFU (High Intensity Focused Ultrasound) concern therapeutic applications [1-4]. Their use in sonochemical processes is as original as it is promising and forms an exciting research area. This paper presents simulations and experimental characterization of sonoreactors and in particular designs for this kind of applications which is quite difficult due to the distortion of the acoustic wave by non linearities of the propagation media or due to the cavitation bubbles disturbance. Depending on the application, one phenomenon can be favored rather than the other. In the case of therapeutic hyperthermy or bulk material irradiation [5], wave distortion allows a faster calorimetric deposit, whereas for sonochemical applications (where free radical species are needed), inertial cavitation is expected. Indeed, cavitation bubble collapses are the place of violent disruption and have been recognized as being essential to enhance chemical reactions. Localization and dynamics of cavitation bubbles induced by HIFU are rather specific but promise to be extremely effective thanks to the concentration at the focal. In the present study, both phenomena were studied (distortion and cavitation) in the case of 2 HIFU transducers designed by IMASONIC. With this in mind, the cavitation zones and the sonochemical active zones are mapped. Measurements carried out by crossing

\footnotetext{
* Corresponding author. Tel.: +33 (0)381662036; fax: +33 (0)381662033

E-mail address: jean-yves.hihn@univ-fcomte.fr (J.-Y. Hihn).
}

different techniques are compared with numerical simulations. Results will contribute to design and scale-up of a new generation of sonoreactors.

\subsection{Experimental details}

Instrumentation: all experiments are performed with two composite HIFU designed by IMASONIC (Besançon, France). The first is a $3 \mathrm{MHz}\left(T_{\mathrm{fc} 3000}\right)$ transducer with a $40 \mathrm{~mm}$ geometrical focal length, while the second operates at a frequency of $750 \mathrm{kHz}$ $\left(T_{\text {fc750 }}\right)$ with a $100 \mathrm{~mm}$ geometrical focal length. Ultrasound are emitted in a cylindrical sonoreactor of Pyrex, (Fig. 1) equipped with a displacement system with four degrees of freedom; three translations guided by micrometric screws and one rotation axis in order to avoid reflections toward the transducer.

Operating procedures: Cavitation bubbles are visualized due to the dispersion of incident laser beam, so that zones with high bubble density appear brighter. An Argon laser Continuum Nd-Yag (532 nm) beam is converted into a vertical static plane sheet of light which enables study of a chosen 2-D zone anywhere in the reactor. Images are collected by a fast camera CCD Sensicam PCO 12 -bit $(1280 \times 1024$ pixels $)$ and give pictures of bubbles [6].

Bubble chemical activity is measured by Sonochemiluminescence (SCL) of Luminol (3-aminophthalhydrazide). Generation of free radicals and hydrogen peroxide by symmetric collapse of inertial bubbles induces oxidation of Luminol molecules which produces emission of a photon ( $430 \mathrm{~nm}$ : blue color). Sonochemiluminescence experiments are carried out with a solution of Luminol 


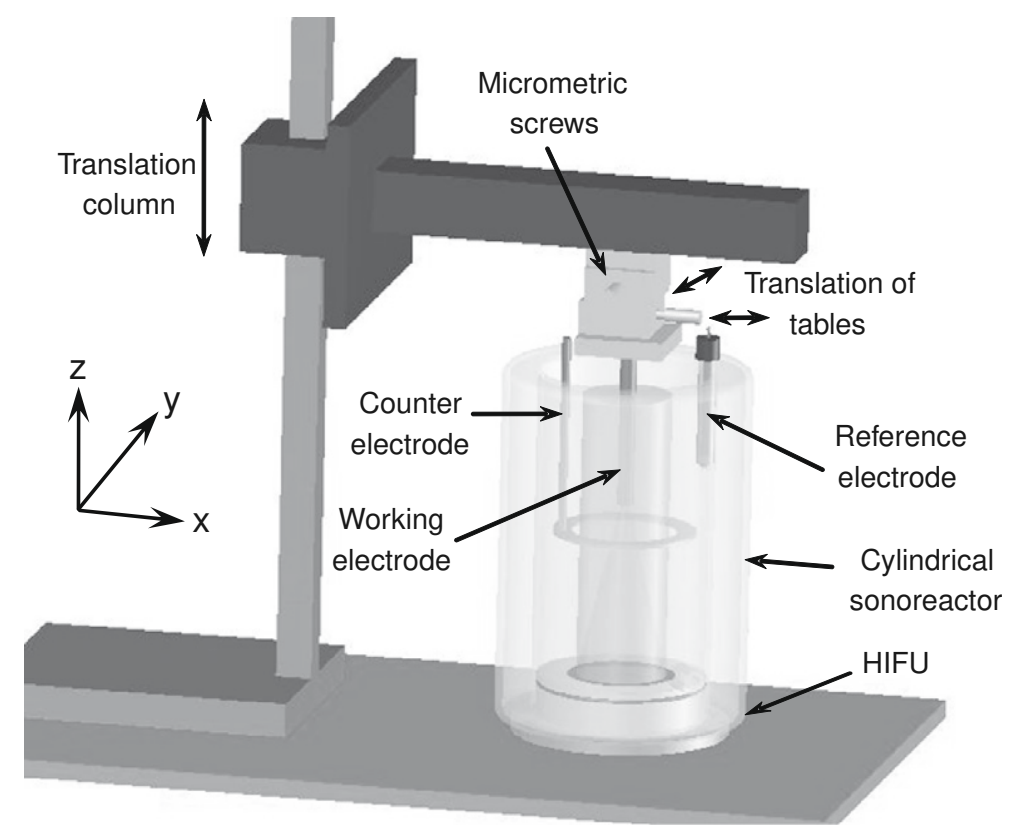

Fig. 1. Experimental set-up.

$(1 \mathrm{mM})$ and $\mathrm{NaOH}(0.1 \mathrm{M})$. Pictures were taken with a digital camera CANON PowerShot G2 (4 Mpixels) placed in the same position for each experiment; shutter speed: $10 \mathrm{~s}$; diaphragm aperture: F2.5; sensibility ISO400. These measures are taken in a lightproof box to avoid disturbance by other light emission. Then, two techniques are used for quantification of sonochemical activity. SCL pictures with Jpeg file extension are post-treated numerically by Matlab ${ }^{\circledR}$ to measure the quantity of photons emitted versus the distance to the transducer. This numerical treatment can be broken down into three steps - subtraction of the SCL picture by a blank in silent conditions in order to eliminate light background - application of a blue filter to the picture in order to enfeeble parasite pixels - counting the pixels with a light intensity higher than a defined threshold. Results are quoted in arbitrary units. Values obtained are an average on a $2 \times 2 \mathrm{~mm}^{2}$ zone centered on the acoustic axis, to allow comparison with the second technique which uses an optical fiber acceptance cone of the same size. A second quantification method consists of displacing an optical fiber in the sonoreactor thanks to micrometric screws. The optical fiber is related to a photon counter Perkin Elmer SPCM-AQR-14 (black noise $100 \mathrm{cps} \mathrm{s}^{-1}$ ) and to an ORTEC MCS-PCI converter card. Acquisition time is equal to camera shutter speed (10 s).

The Fricke dosimetry method allows quantification of production of free radicals and hydrogen peroxide produce by homolytic scission of water molecules due to inertial cavitation collapse [6,7]. The Fricke solution consists of $\mathrm{Fe}\left(\mathrm{SO}_{4}\right)_{2}\left(\mathrm{NH}_{4}\right)_{2} \cdot 6 \mathrm{H}_{2} \mathrm{O}(0.25 \mathrm{mM})$, $\mathrm{H}_{2} \mathrm{SO}_{4}(0.4 \mathrm{M})$ and $\mathrm{NaCl}(1 \mathrm{mM})$. The sampling absorbance $(304 \mathrm{~nm})$ is measured by UV spectrometer (HITACHI U-2001 ${ }^{\circledR}$ ), the concentration of solution in $\mathrm{Fe}^{3+}$ is determined as a function of absorbance. The sonochemical yield $(G)$ has been calculated according to the Iida equation $G\left(\mathrm{Fe}^{3+}\right)=\left[\mathrm{Fe}^{3+}\right] \cdot V_{T} \cdot E_{a}^{-1}$, where $E_{a}$ is the acoustic energy $(\mathrm{J})$ and $V_{T}$ the volume of solution $\left(\mathrm{dm}^{3}\right)$ [7].

\section{Results and discussion}

\subsection{Modeling the HIFU acoustic field}

Prior to any experimental characterization, the acoustic field was modeled by Rayleigh's equation to describe the theoretical focalization properties and pressure distribution specific to each transducer (Ultrasim module of Matlab ${ }^{\circledR}$ developed at the University of Oslo). Table 1 shows the simulation results of pressure gain in focal areas for both transducers $\left(T_{\mathrm{fc} 750}\right.$ and $\left.T_{\mathrm{fc} 3000}\right)$. Fig. 2a shows detail of the pressure field gain in the vicinity of the focal for $T_{\mathrm{fc} 750}$ $(z$ - axis is the axial distance of the transducer and $x$-axis is the radial direction). It appears that depth of field as well as focal width (boundary $-3 \mathrm{~dB}$ ) are very large (respectively 46 and $3.7 \mathrm{~mm}$ ). The maximum acoustic pressure gain which corresponds to the ratio between acoustic pressure on the emitted surface and acoustic pressure at the focal is about 10.5 and located at $90 \mathrm{~mm}$. This difference is common for this kind of HIFU (long focal length and low frequency), because of the competition between geometrical focalization of the beam and its natural divergence. For the $T_{\mathrm{fc} 3000}$ (Fig. 2b), the depth of field and the focal width are significantly smaller (respectively 3.3 and $0.5 \mathrm{~mm}$ ), according to a higher focalization gain: 67.3. Fig. 3 shows the spatial distribution of the $T_{\mathrm{fc} 3000}$ pressure gain for the whole acoustic field. Several specificities can be observed as an important peak of pressure gain at the focal and as a more intense gain on the envelope of the distribution cone. Local cancellations of the gain on the acoustic axis are observed and assigned to destructive interferences.

\subsection{Measurement by hydrophone of wave shape at the focal}

The shape of the pressure wave of the $T_{\mathrm{fc} 3000}$ has been measured with a $3 \mathrm{MHz}$ hydrophone (Imasonic) to check its distortion. Indeed in event of high power wave propagation, local pressure variations lead to non linearities that result in by wave velocity

Table 1

Technical characteristics of HIFU

\begin{tabular}{lll}
\hline Transducer & $T_{\mathrm{fc} 750}$ & $T_{\mathrm{fc} 3000}$ \\
\hline Frequency $(\mathrm{kHz})$ & 750 & 3 \\
Active diameter $(\mathrm{mm})$ & 50 & 40 \\
Active surface $\left(\mathrm{cm}^{2}\right)$ & 19.96 & 13.47 \\
Geometric focal $(\mathrm{mm})$ & 100 & 40 \\
Acoustic focal $(\mathrm{mm})$ & 90 & 39.9 \\
Diameter of focal area $-3 \mathrm{~dB}(\mathrm{~mm})$ & 3.71 & 0.5 \\
Depth of field $-3 \mathrm{~dB}(\mathrm{~mm})$ & 46 & 3.3 \\
Focus gain in intensity & 110 & 4540 \\
Focus gain in pressure & 10.5 & 67.3 \\
\hline
\end{tabular}



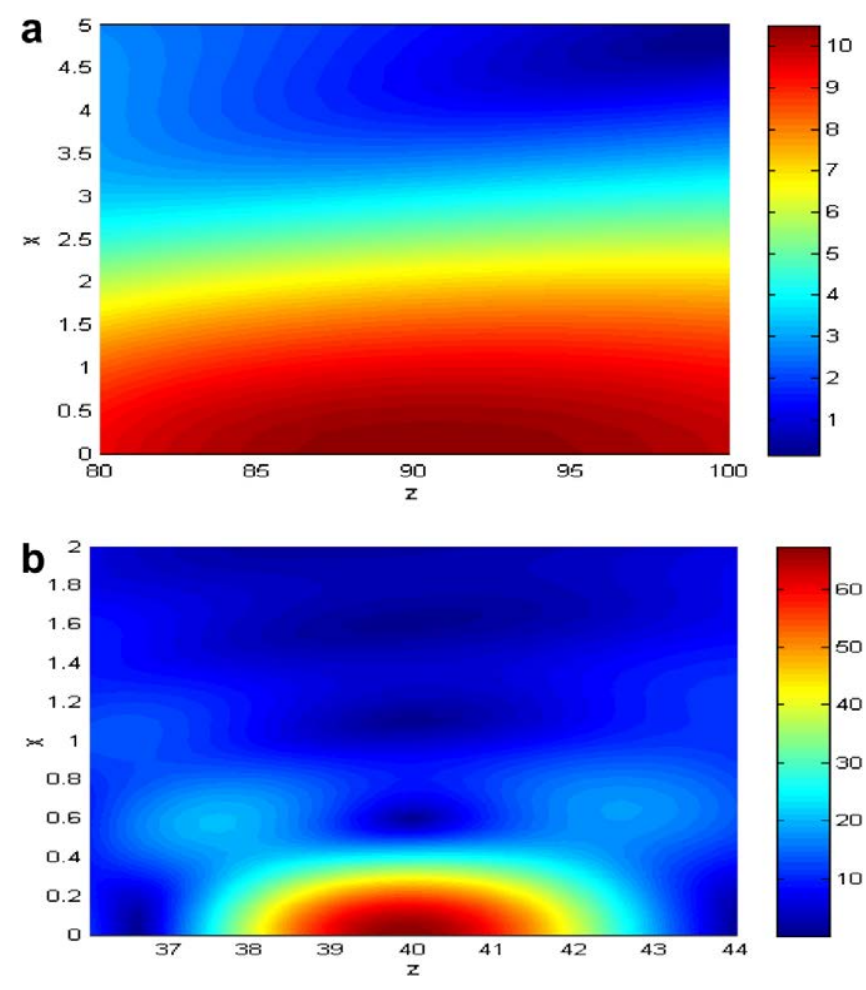

Fig. 2. (a) Acoustic pressure gain $\left(p_{\mathrm{ac}} / p_{0}\right)$ in the focal area of the $T_{\mathrm{fc} 750}$ (situated at $z=0)$. (b) Acoustic pressure gain $\left(p_{\mathrm{ac}} / p_{0}\right)$ in the focal area of the $T_{\mathrm{fc} 3000}$ (situated at $z=0)$.

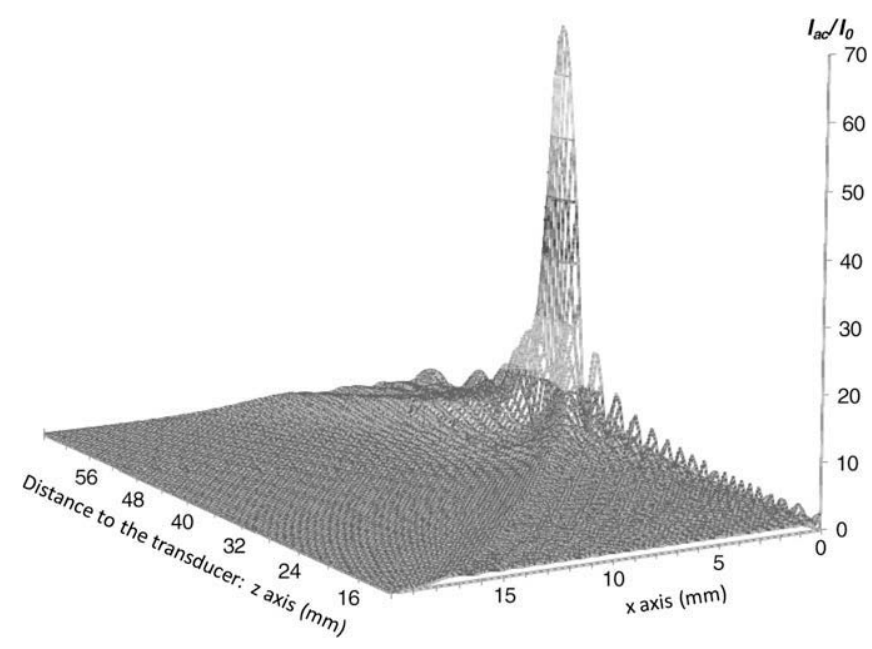

Fig. 3. Acoustic intensity gain $\left(I_{\mathrm{ac}} / I_{0}\right)$ in the acoustic field of the $T_{\mathrm{fc} 3000}$.

variations. High pressure zones travel faster than low pressure zones and the sinusoidal shape of the wave, observed for powers lower than $1 \mathrm{~W}$ (Fig. 4a), tends toward a shock wave shape (Fig. $4 \mathrm{~b}$ and c). This distortion generates harmonics ( $2 f, 3 f \ldots$ ) which can be seen on the spectrum analysis (Fig. 4). The acoustic energy transported by the fundamental frequency is shifted towards harmonic frequencies. For powers higher than $0.3 \mathrm{~W}$, all the energy $\left(E_{0}\right)$ which was transported at the fundamental frequency $E_{2.5 \mathrm{MHz}}=E_{0}$ moves to harmonic frequencies as follows:

$E_{2.5 \mathrm{MHz}}+E_{5 \mathrm{MHz}}+E_{7.5 \mathrm{MHz}}+E_{10 \mathrm{MHz}}=E_{0}$ then $\frac{E_{2.5 \mathrm{MHz}}}{E_{0}}$

1

$=\frac{1}{1+k_{5 \mathrm{MHz}}+k_{7.5 \mathrm{MHz}}+k_{10 \mathrm{MHz}}}$ where $k_{5 \mathrm{MHz}}=\frac{E_{5} \mathrm{MHz}}{E_{2.5 \mathrm{MHz}}}$ which is determined with the spectrum analysis.

For $10.6 \mathrm{~W}, \frac{E_{2.5 \mathrm{MHz}}}{E_{0}}=0.973$ and for $75.4 \mathrm{~W}, \frac{E_{2.5 \mathrm{MHz}}}{E_{0}}=0.743$.

This phenomenon induces higher absorption of the wave by the propagation media because the absorption coefficient depends on frequency [8] and can contribute to a focal shift in transducer direction.

\section{Localization and behavior of cavitation bubbles}

\subsection{Low powers: stationary bubbles}

In event of low power ultrasound or presence of rigid boundaries (reflector located in the propagation field), a standing wave system takes place in the sonoreactor. In the first moment of irradiation, cavitation bubbles appear at the focal or are arranged into pressure antinodes (Fig. 5) and stay in the same arc of circle. They are generated, then grow, coalesce or collapse within this defined location (Fig. 6a-c) and finally form clusters of large bubbles (Fig. 7). Stable bubbles (shown in the pictures) as well as inertial bubbles, highlighted by SCL can be observed. After a few milliseconds, these large bubbles or clusters coalesce in a perpendicular direction (Fig. 6d and e). Thanks to the action of the secondary Bjerknes force generated by the pressure field radiated from pulsating bubbles, they are thrown in the wave direction (Fig. 6f). The "traveling bubbles" [9] located in the prefocal zone (Fig. 7ac) reach the focal area and form a very large cluster, which becomes increasingly larger with time (Fig. 7d-f). Displacement of the bubbles inside the standing wave system is attributed to the primary Bjerknes force generated by the pressure gradient [10] and the acoustic streaming which act on bubbles.

For specific use of HIFU in surface chemistry, a target is irradiated by focusing the acoustic beam on the surface as in the case of therapeutic applications. As the material is rigid and acts as a reflector, a bubble cloud is observed close to the surface. This cluster grows and takes a hemispherical shape (Fig. 8). This intense cavitational activity causes major damage to the material surface, observed in previous experiments and usually attributed to asymmetric bubble collapse [11]. Chen et al. working with smoother substrates observe the same kind of alteration on the surface of porcine liver tissue [4]. Tracking sonochemical activity distribution, the SCL pictures show the zone where hydrogen hydroxyl production is higher. In Fig. 9a, the transducer is situated on the left side of the picture and the target presents an incident angle of $30^{\circ}$ with the acoustic axis. Surprisingly, the more intense SCL emissions are situated after the focal located directly at the surface, in the wave reflected by the target. This was not expected because few cavitation bubbles are detected by tomographic pictures in this part of the acoustic field (Fig. 9b). On the contrary, a dark zone appears upstream, whereas a large quantity of large cavitation bubbles can be seen at the same location by tomographic pictures recorded in the same conditions in Fig. 8b. A direct relationship should not be systematically implied between the SCL and the detection of cavitation bubbles by tomography because they are not necessarily active in the sonochemical point of view.

\subsection{High powers: moving bubbles}

When higher powers are applied to our transducers or in the specific case of free interface irradiation or pulsed emission, behavior of the cavitation bubbles completely changes. It is impossible to observe the establishment of standing waves and the bubbles are no longer located in restricted areas. Rather they follow a global movement in the propagation wave direction in the reactor axis with recirculation flows at the walls (Fig. 10). This difference stems 

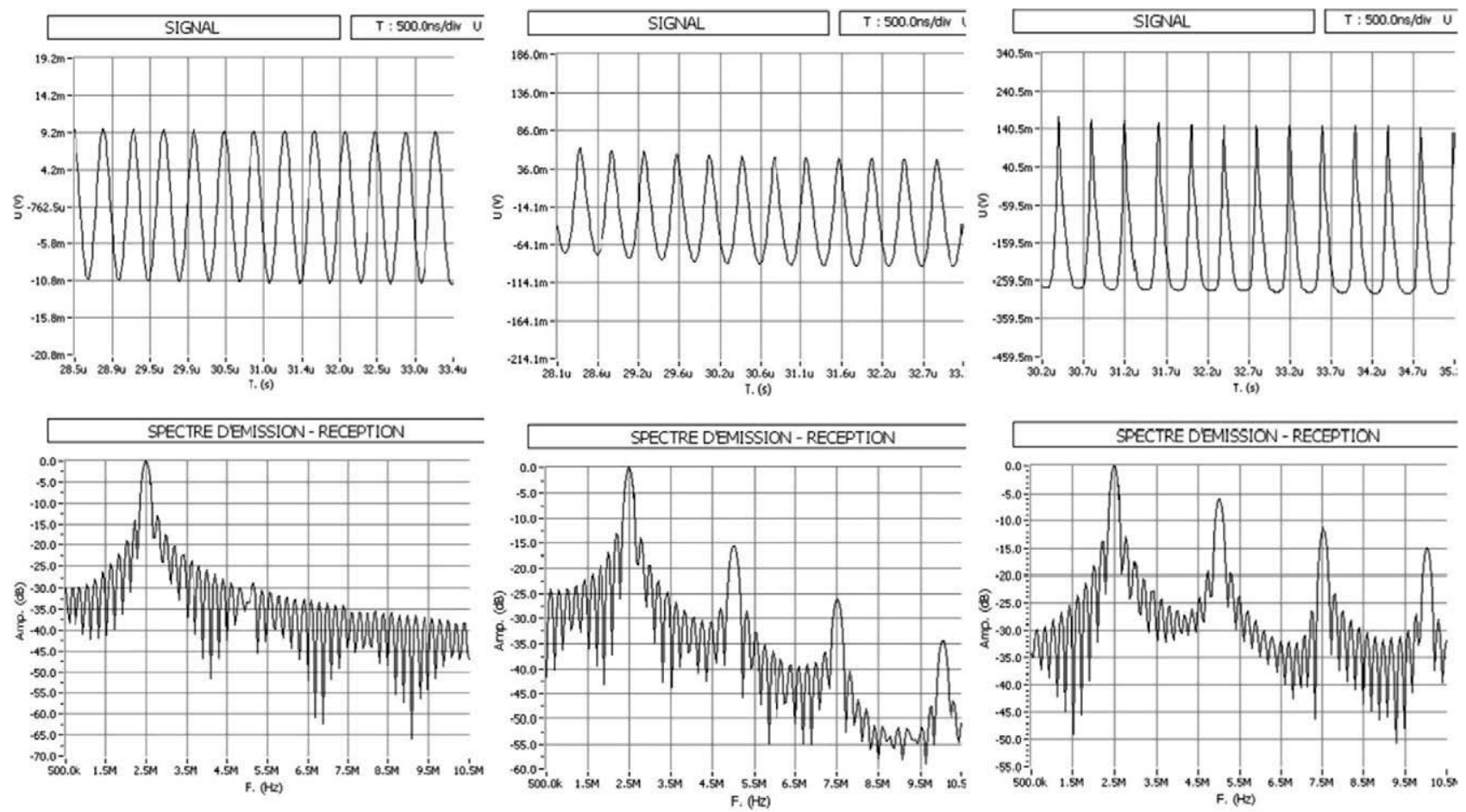

(a) Electrical power $0.3 \mathrm{~W}$

(b) Electrical power $0.3 \mathrm{~W}$

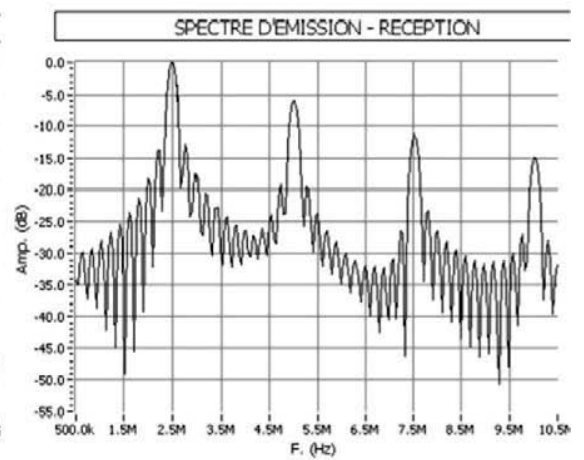

(c) Electrical power $0.3 \mathrm{~W}$

Fig. 4. Shape wave at the focal of $T_{\mathrm{fc} 3000}$ for different electrical powers.

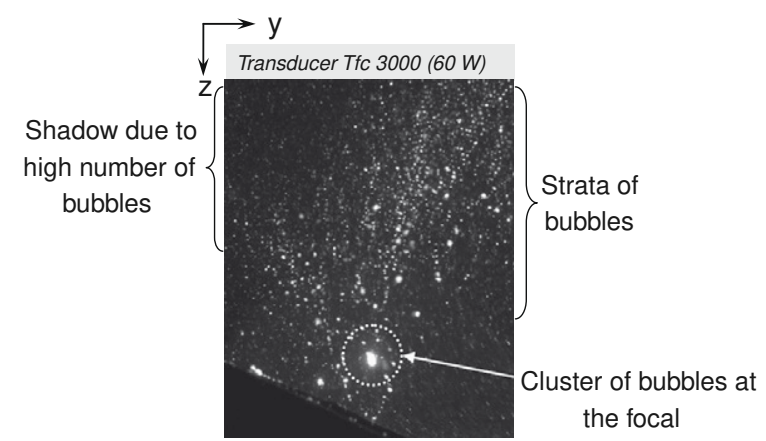

Fig. 5. Cavitation field of $T_{\mathrm{fc} 3000}$ in the whole acoustic field (60 W).

from breakdown of the levitation equilibrium by an increase in all perturbations applied to the bubble, such as Bjerknes forces and acoustic streaming. Therefore, the sonochemical interest of the reactor is radically different since energy is transported throughout the reactor while it is trapped in the pressures nodes in the case of a standing wave system. Bubbles located upstream from the focal are numerous and very small in size, resembling a dust cloud. When they cross the focal, bubbles coalesce and finally form long trails grouped on the propagation cone outer layer, whereas the post-focal zone directly on the wave axis has fewer bubbles. We can note here the major advantage of acquiring tomographic pictures obtained by 2-D laser sheets of light to describe more accurately bubble behavior in the axial cross section, whereas classic lighting will only allow the outer layer to be viewed. This weak bubble concentration can be linked to the decrease in acoustic pressure due to attenuation by the numerous bubbles at the focal, which play the role of a reflector. Sonochimiluminescence measurements are also taken with high ultrasonic powers and a free water/air interface so that sonochemical activity can be localized, i.e. free radical generation in a system in global movement. Pictures were recorded for increasing powers (Fig. 11) and the general shape of the SCL is conical as expected thanks to the simulation (Fig. 3). At low powers, cavitation activity is reduced and focused just below the focal, with only two trails of light beyond. In this case, global intensity increases with power, and beyond $30 \mathrm{~W}$ active zones grow on either side of the focal. Nevertheless, an absence of SCL can be noted in the focal vicinity as well as in the downstream acoustic axis and in the neighborhood of the trans-

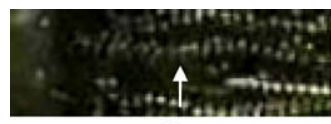

a

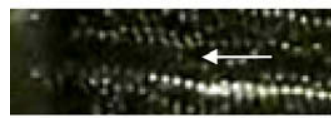

d

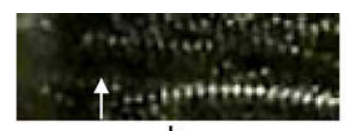

b

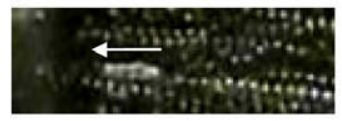

e

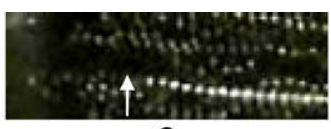

C

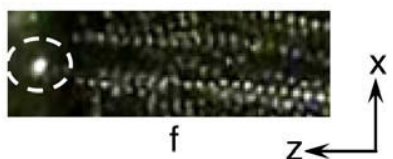

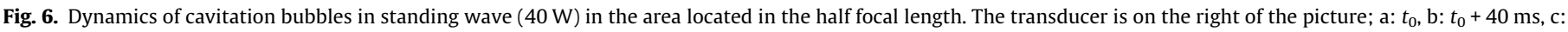
$t_{0}+80 \mathrm{~ms}$, d: $t_{0}+120 \mathrm{~ms}$, e: $t_{0}+160 \mathrm{~ms}$, f: $t_{0}+200 \mathrm{~ms}$. 


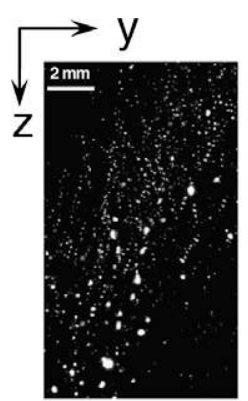

a

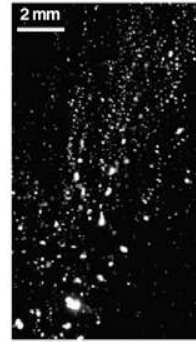

b

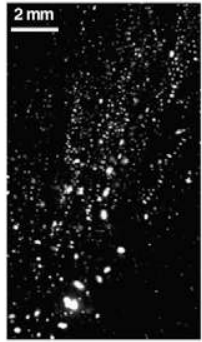

C

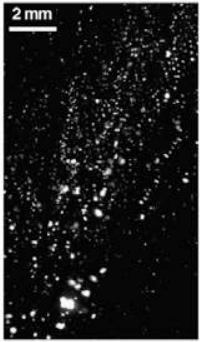

d

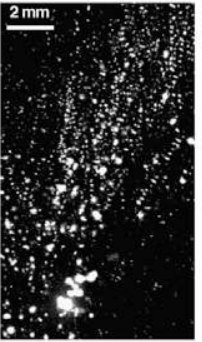

e

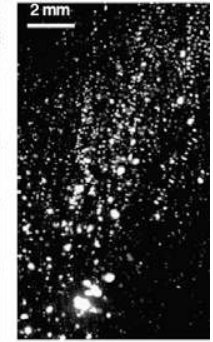

f

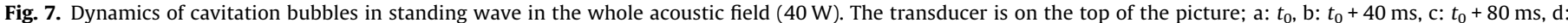
$t_{0}+120 \mathrm{~ms}$, e: $t_{0}+160 \mathrm{~ms}$, f: $t_{0}+200 \mathrm{~ms}$.

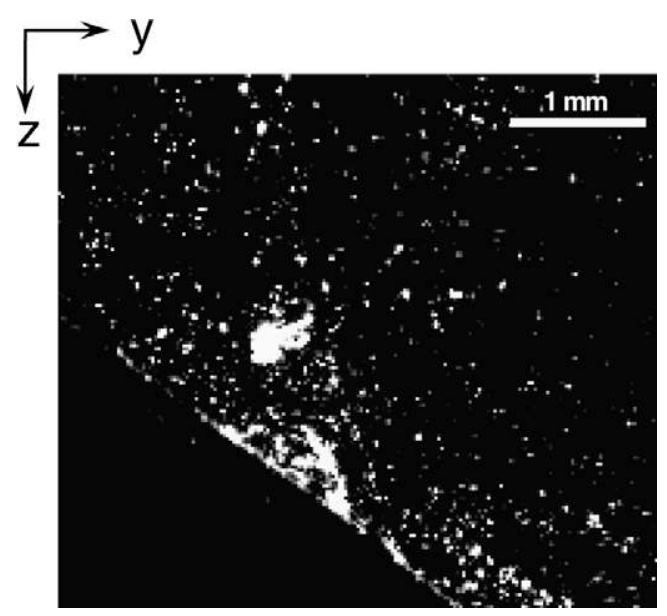

Fig. 8. Hemispherical cluster of bubbles in the neighborhood of a rigid surface $(40 \mathrm{~W})$.
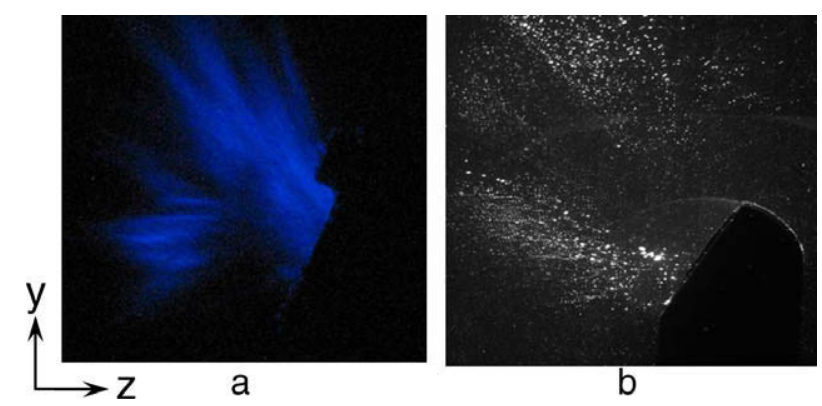

Fig. 9. SCL and tomography at $40 \mathrm{~W}$, irradiation of a rigid surface.

ducer. This point is not surprising for the zones located close to the emitting surface or the downstream one because of the few bubbles present in the tomographic pictures. Both zones correspond to well-known low levels of acoustic energy, the first because focalization and therefore energy gain are not sufficient to induce cavitation, and the second due to the energy losses induced by bubble density at the focal. On the contrary, it is more difficult to give an obvious explanation for SCL quenching at the focal because at the same time, a high concentration of bubbles is observed.

\section{Quantification of cavitation bubble activity}

To complete the HIFU reactor study, quantification of sonochemical activity was conducted with the $T_{\text {fc750. The efficiency }}$ yield measurements obtained by Fricke's dosimetry estimate

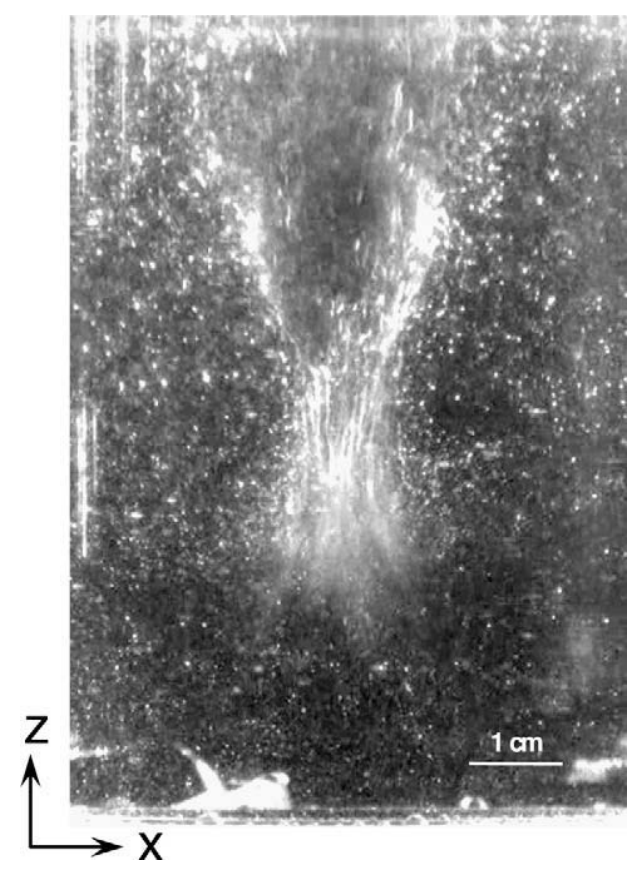

Fig. 10. Dynamics of cavitation bubbles for high power irradiation $(40 \mathrm{~W})$. The transducer $T_{\mathrm{fc} 3000}$ is at the bottom of the picture.

chemical reactivity of the inertial cavitation bubbles in the whole reactor. In all cases, yield is virtually constant whatever the transmitted power $(8.3 \pm 0.5) \times 10^{-10} \mathrm{~mol} \mathrm{~J}^{-1}$. This ‘saturation' phenomenon has been previously observed for flat transducers by Mark et al. [12] who found a maximum of $35 \times 10^{-10} \mathrm{~mol} \mathrm{~J}^{-1}$ at $300 \mathrm{kHz}$. Iida et al. [7] measured a sonochemical yield of $2.7 \times 10^{-10} \mathrm{~mol} \mathrm{~J}^{-1}$ at $130 \mathrm{kHz}$ and Mandroyan et al. [6] gives values between 5 and $10 \times 10^{-10} \mathrm{~mol} \mathrm{~J}^{-1}$ at 20 and $40 \mathrm{kHz}$. Koda et al. [13] summarizes all these results as a function of frequency and gives values ranging between 2.3 and $3.7 \times 10^{-10} \mathrm{~mol} \mathrm{~J}^{-1}$ at low frequencies (20 to $45 \mathrm{kHz}$ ) and around $20 \times 10^{-10} \mathrm{~mol} \mathrm{~J}^{-1}$ at frequencies of 100 and $500 \mathrm{kHz}$. They noticed a strong decrease for higher frequencies $\left(2.6 \times 10^{-10} \mathrm{~mol} \mathrm{~J}^{-1}\right.$ at $\left.1.2 \mathrm{MHz}\right)$. We can affirm therefore that the sonochemical output of the $750 \mathrm{kHz}$ HIFU is better than for a low-frequency transducer. On the other hand, it is possible to assure that the HIFU are on average less sonochemically active than the plane transducers at the same frequency. We note nevertheless that the volume radiated by a HIFU is already smaller than that of a plane transducer for the same diameter. Looking at the sonochemical output to the radiated theoretical volume ratio, a value of $22.1 \times 10^{-10} \mathrm{~mol} \mathrm{~J}^{-1}$ is determined, i.e. close to those given in the literature. Even if the Fricke dosimetry gives a large 
range of magnitude for sonoreactor activity, the need for another technique allowing local counting of radical species concentration in the relevant acoustic zone still exists.

Since the light emitted by SCL is proportional to the radical species concentration, two methods have been designed to take mea- surements at different locations in the reactor. The first method is based on pixel counting on SCL pictures (indirect counting after camera recording) and the second method on counting of the number of emitted photons (direct counting using a photon counter and an optic fiber). Both methods were used and compared to
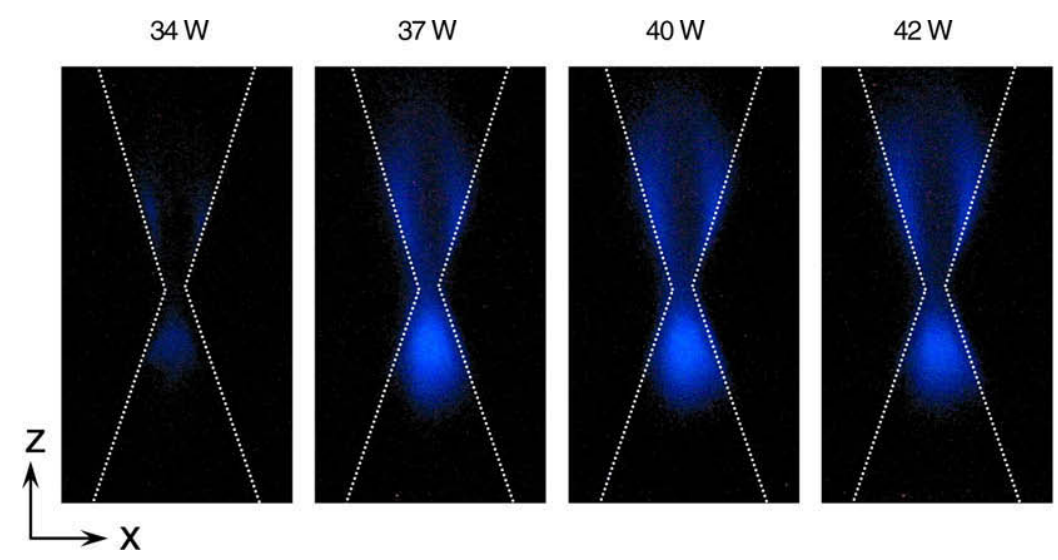

$46 \mathrm{~W}$

Fig. 11. SCL for different powers, irradiation of a free surface.
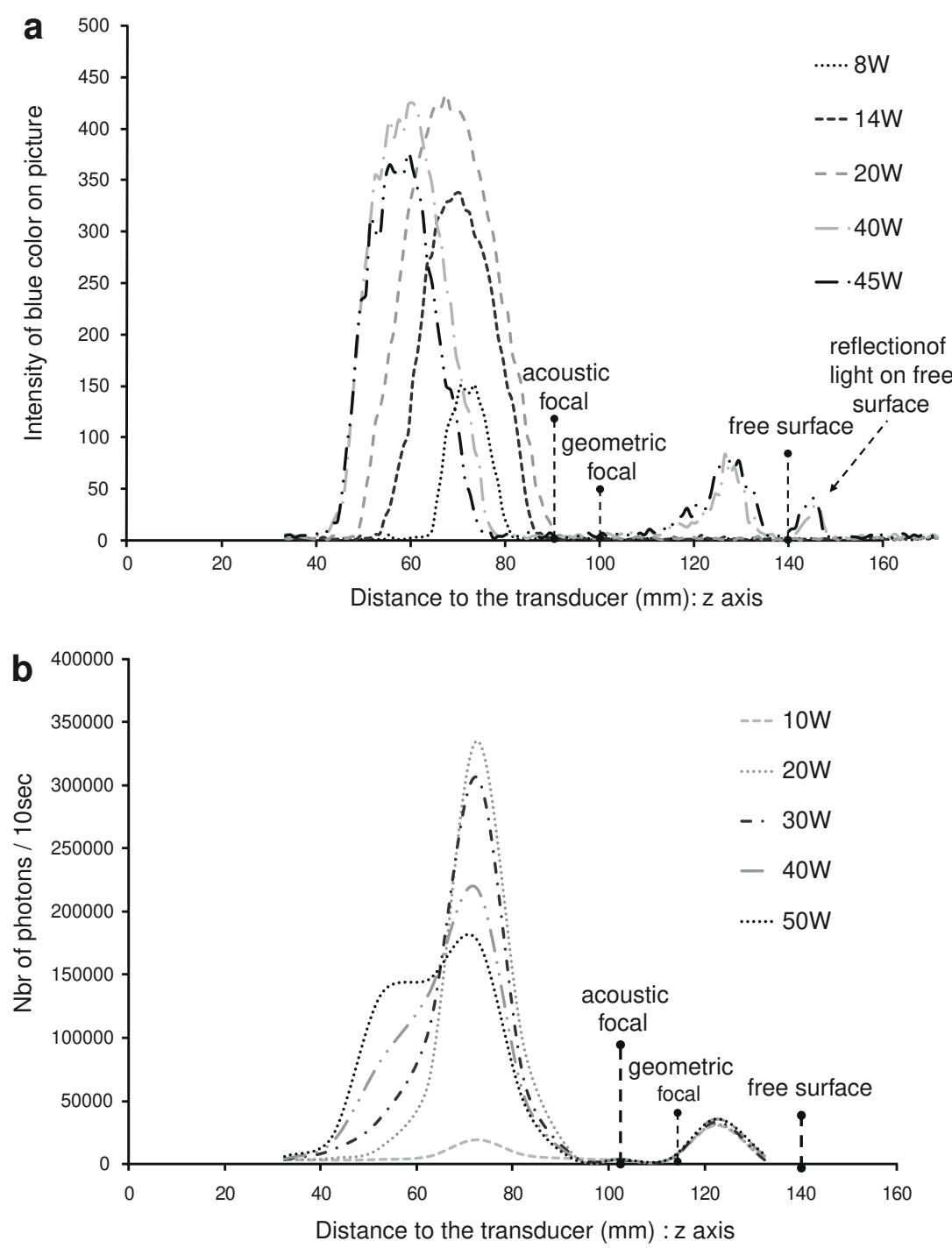

Fig. 12. (a) Pixel counting of SCL pictures. (b) Photon counting with optic fiber. 


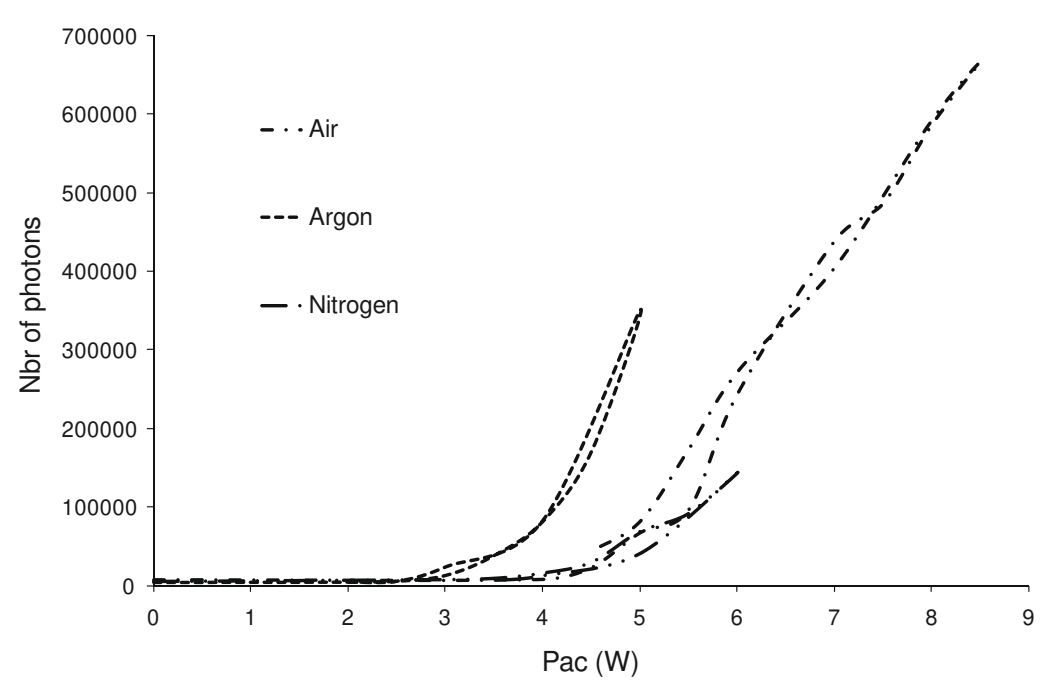

Fig. 13. SCL threshold with $T_{\mathrm{fc} 750}$ for different saturated gases (air, nitrogen, argon).

avoid any risk of possible saturation of camera CCD sensors and to fill the rigidity of the photon counter measurement cone. Results obtained on the propagation abscissas are presented in Fig. 12a (pixel counting) and Fig. 12b (photon counting). Fig. 12 shows the appearance of SCL at $10 \mathrm{~W}$ for both quantification methods in a zone centered on $73 \mathrm{~mm}$ of the emitting surface, slightly upstream from the acoustic focal. SCL intensity increases as power increases, to reach a peak of $30 \mathrm{~W}$ in the case of pixel counting in Fig. 11a. Then, SCL intensity decreases in this zone and enlarges in the transducer direction. For powers higher than $40 \mathrm{~W}$, SCL appears in the post-focal zone. For photon counting, the general shape remains the same, with better proportionality between the power and the maximum observed at $73 \mathrm{~mm}$ abscissa (Fig. 12b). In both cases, it is possible to confirm the absence of SCL directly at the focal and in the post-focal zone, immediately downstream from the propagation axis. Several assumptions can be made concerning SCL quenching in these zones, particularly by taking into account previous works on sonoluminescence and SCL $[10,14-$ 16]. They observed sudden quenching of the SCL at excessive ultrasonic intensity, as bubble behavior clearly changes. Bubble filament organization changes into clusters due to the secondary Bjerknes forces and therefore occurs away from the pressure antinodes [13], with the result that the collapse releases less energy. Nevertheless, gentle agitation is sufficient to suppress quenching in their case and to observe again a good level of SCL. In our case, i.e. a not established standing wave system, an additional assumption can be made. First, the high bubble concentration in this zone (Fig. 10) will certainly favor interactions as in the case of collapse in the surface vicinity. On the other hand, the high velocities occurring at the focal and immediately downstream will lead to bubble deformation due to viscous frictions. In both cases, the collapse will be asymmetric and therefore insufficient for free radical production. Another contribution of the photon counting technique is determination of the SCL threshold which is directly linked to inertial cavitation appearance. Pure water is saturated by bubbling argon, nitrogen or air and the optic fiber end is located at a distance of $73 \mathrm{~mm}$ from the transducer, in the zone where the beginning of light emission was previously observed. Photon counting starts in silent conditions, then sonication begins up to a few watts over the light detection threshold, and reversibility is checked by decreasing power (Fig. 13). For all gases, light emission remains at its background level, then increases regularly over a defined break point. The reverse ways are close and the limits where the curves join the background levels are exactly the same, dismissing any hysteresis effect. Threshold values are identical for air and nitrogen, i.e. an input of $4 \mathrm{~W}$ (corresponding to $0.812 \mathrm{MPa}$ at the focal) and slightly lower for argon, i.e. an input of $2.8 \mathrm{~W}$ (corresponding to $0.68 \mathrm{MPa}$ at the focal), following the gas solubilities which are: $\arg 0 n$ 0.04 L/L $\gg$ air $0.0187 \mathrm{~L} / \mathrm{L} \geqslant$ nitrogen $0.01542 \mathrm{~L} / \mathrm{L}$.

\section{Conclusion}

Use of HIFU for sonochemical reactors is a completely different approach than for therapeutic applications, because continuous excitation leads to bubble production and circulation in the reactor which changes its behavior. Tomography measurements show that the cavitation bubbles generated by HIFU are mainly located on the outer layer of the propagation cone in the post-focal zone, but with a specific distribution highlighted by the 2-D pictures which allow low activity zones to be discovered. Greatest acoustic activity expected by simulation is not located at the geometrical focal, but is confirmed by a high concentration of bubbles. On the contrary, the main sonochemical activity shifts slightly toward the transducer, whereas quenching of inertial cavitation is observed directly at the focal. Finally, SCL thresholds have been determined for argon from one side for only $2.8 \mathrm{~W}$ of input power and approximately $4 \mathrm{~W}$ for air and nitrogen from another side.

\section{Acknowledgments}

The authors would like to thank gratefully the "région FrancheComté" for the PhD grant and the DRIRE (French ministry for industry) for the financial support in the frame of the "ULTRASUR Project" (pôle de compétitivité Microtechniques).

\section{References}

[1] H. Chen, X. Li, M. Wan, The inception of cavitation bubble clouds induced by high-intensity focused ultrasound, Ultrasonics 44 (2006) e427-e429.

[2] H. Chen, X. Li, M. Wan, Spatial-temporal dynamics of cavitation bubble clouds in $1.2 \mathrm{MHz}$ focused ultrasound field, Ultrasonics Sonochemistry 13 (2006) 480-486.

[3] H. Chen, X. Li, M. Wan, S. Wang, High-speed observation of cavitation bubble cloud structures in the focal region of a $1.2 \mathrm{MHz}$ high-intensity focused ultrasound transducer, Ultrasonics Sonochemistry 14 (2007) 291-297.

[4] H. Chen, X. Li, M. Wan, S. Wang, High-speed observation of cavitation bubble clouds near a tissue boundary in high-intensity focused ultrasound fields, Ultrasonics 49 (2009) 289-292.

[5] L. Hallez, F. Touyeras, J.Y. Hihn, J. Klima, Energetic balance in an ultrasonic reactor using focused or flat high frequency transducers, Ultrasonics Sonochemistry 14 (2007) 739-749. 
[6] A. Mandroyan, R. Viennet, Y. Bailly, M.L. Doche, J.Y. Hihn, Modification of the ultrasound induced activity by the presence of an electrode in a sonoreactor working at two low frequencies ( 20 and $40 \mathrm{kHz}$ ). Part I: active zone visualization by laser tomography, Ultrasonics Sonochemistry 16 (2009) 88-96.

[7] Y. Iida, K. Yasui, T. Tuziuti, M. Sivakumar, Sonochemistry and its dosimetry, Microchemical Journal 80 (2005) 159-164.

[8] R.L. Clarke, G.R. Ter Haar, Production of harmonics in vitro by high-intensity focused ultrasound, Ultrasound in Medicine \& Biology 25 (1999) 1417-1424.

[9] R. Mettin, A.A. Doinikov, Translational instability of a spherical bubble in a standing ultrasound wave, Applied Acoustics 70 (2009) 1330-1339.

[10] S.-I. Hatanaka, H. Mitome, K. Yasui, S. Hayashi, Multibubble sonoluminescence enhancement by fluid flow, Ultrasonics 44 (2006) e435-e438.

[11] M.L. Doche, J.Y. Hihn, F. Touyeras, J.P. Lorimer, T.J. Mason, M. Plattes, Electrochemical behaviour of zinc in $20 \mathrm{kHz}$ sonicated $\mathrm{NaOH}$ electrolytes, Ultrasonics Sonochemistry 8 (2001) 291-298.
[12] G. Mark, A. Tauber, R. Laupert, H.-P. Schuchmann, D. Schulz, A. Mues, C. Von Sonntag, OH-radical formation by ultrasound in aqueous solution - part II: terephthalate and Fricke dosimetry and the influence of various conditions on the sonolytic yield, Ultrasonics Sonochemistry 5 (1998) 41-52.

[13] S. Koda, T. Kimura, T. Kondo, H. Mitome, A standard method to calibrate sonochemical efficiency of an individual reaction system, Ultrasonics Sonochemistry 10 (2003) 149-156.

[14] S.-I. Hatanaka, K. Yasui, T. Kozuka, T. Tuziuti, H. Mitome, Influence of bubble clustering on multibubble sonoluminescence, Ultrasonics 40 (2002) 655660.

[15] H. Mitome, S. Hatanaka, Anomalous changes of sonochemical luminescence intensity under stirring operation, Journal of the Acoustical Society of America 108 (2000) 2494.

[16] H. Mitome, S.I. Hatanaka, Optimization of a sonochemical reactor using a pulsing operation, Ultrasonics 40 (2002) 683-687. 